\title{
Comportement des rideaux de palplanche : expérimentation en vraie grandeur et prédictions numériques
}

\section{SHAHROUR}

S. GHORBANBEIGI

Laboratoire de Mécanique de Lille (URA 1441)

École Centrale de Lille BP 48

59651 Villeneuve d'Ascq Cedex

\section{P.A. von WOLFFERSDORFF}

Institut für Bodenmechanik und Flesmechanik Lehrstuhl für Bodenmechanik und Grundbau

Universität Karlsruhe Engler-Bunte-Ring, Postfach 6980 D-76128 Karlsruhe
Dans cet article, on présente les résultats des prédictions numériques d'une expérimentation en vraie grandeur réalisée sur un rideau de $7 \mathrm{~m}$ de largeur fíché dans un sol sableux à $6 \mathrm{~m}$ de profondeur. L'expérimentation a comporté diverses phases de chargement: excavation, butonnage, reprise de l'excavation, chargement en surface et réduction de la longueur des butons jusqu'à l'état-limite. La modélisation numérique a été effectuée avec un modèle élastique parfaitement plastique utilisant le critère de Mohr-Coulomb et une règle d'écoulement non associée. Après une description de la démarche suivie pour la modélisation (détermination des paramètres, schématisation des conditions aux limites et du chargement, discrétisation), on présente une confrontation des résultats numériques à ceux de l'expérimentation. Afin de bien cerner la précision des prédictions numériques, on donne les résultats d'une étude de l'influence de principaux paramètres mécaniques sur la simulation numérique de l'expérimentation.

\section{Behaviour of sheet piles: field test and numerical predictions}

This paper includes a presentation of the results of numerical predictions of a sheetpile wall field test performed in a sandy soil. The loading program included: excavation, installation of struts, continuation of the excavation, application of a surface loading, and the reduction of the struts length until the reach of the limit state. Numerical predictions were performed using an elastic - perfectly plastic constitutive relation with the MohrCoulomb criterion and a non associated flow rule. After a description of the methodology followed in numerical modelling (identification of constitutive parameters, modelling of boundary conditions and discretization), we present a comparison of numerical results to those of the experimentation. In order to investigate the accuracy of numerical predictions, we give the results of a parametric study on the influence of constitutive parameters on numerical simulations. 


\section{1}

\section{Introduction}

Les rideaux de palplanche sont utilisés dans divers types d'ouvrages: blindage de fouilles en site urbain, batardeaux, rideaux d'étanchéité et murs de quai. Leur calcul en déformation constitue un problème fort complexe d'interaction sols-structures qui fait intervenir le comportement des sols, la rigidité des rideaux, les phases des travaux, les conditions d'ancrage et les conditions hydrauliques.

Le calcul de structures comportant des rideaux peut être effectué à l'aide de deux méthodes, à savoir la méthode aux coefficients de réaction et la méthode des éléments finis. Dans la première approche, le sol est schématisé par des ressorts dont le comportement est généralement modélisé par un modèle élastique parfaitement-plastique. La grande difficulté de l'utilisation de cette approche réside dans la détermination des paramètres mécaniques qui dépendent sensiblement, en plus de la nature des sols, de l'interaction sol-rideau et des conditions d'ancrage [1], [2]. Ajoutons à cela les difficultés de l'utilisation de cette approche dans les problèmes faisant intervenir des interactions avec la nappe ou avec des ouvrages existants. Dans l'approche éléments finis, le sol est modelisé comme un milieu continu dont le comportement peut être décrit à l'aide de divers modèles de comportement dont les paramètres peuvent être déterminés indépendamment des conditions d'interaction rideau-sol ou d'ancrage. Elle permet de traiter des problèmes complexes faisant intervenir la présence d'eau ou des interactions avec d'autres ouvrages.

L'utilisation de ces approches pour le calcul de rideau de soutènement nécessite des vérifications sur des expérimentations en vraie grandeur. Conscient de cette nécessité, 1'Université de Karlsruhe a réalisé une expérimentation sur un rideau de $7 \mathrm{~m}$ de largeur fiché dans un sol sableux à $6 \mathrm{~m}$ de profondeur [6]. L'expérimentation a comporté diverses phases de chargement:

(a)

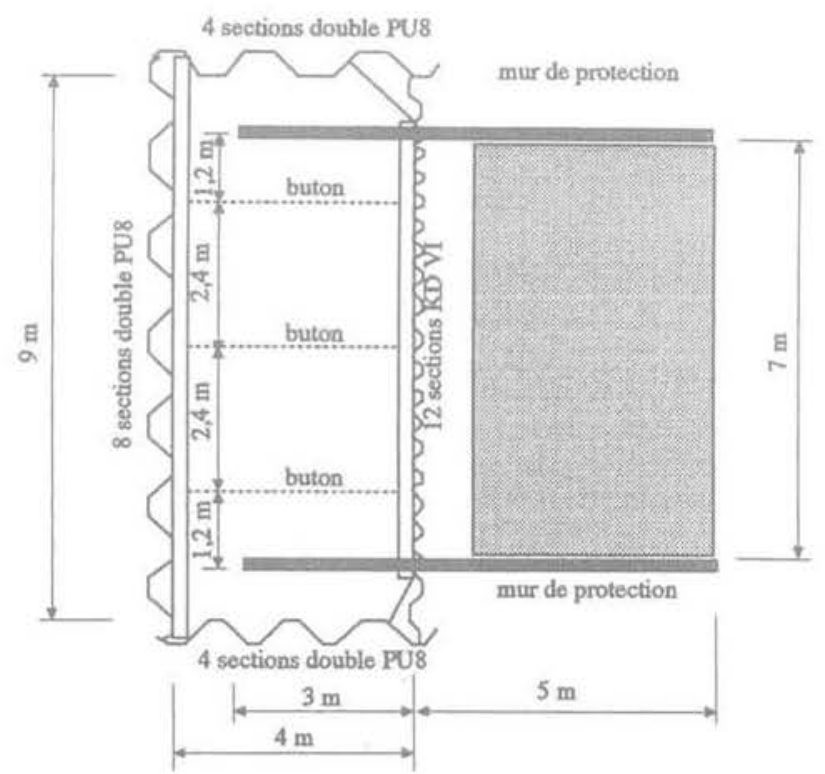

FIG.1 Dispositif d'expérimentation. Experimentation set up. excavation, butonnage, reprise de l'excavation, chargement en surface et réduction de la longueur des butons jusqu'à l'état-limite. L'essai a été bien instrumenté avec des mesures de la déformée du rideau, des forces dans les butons, des tassements en surface et de la pression appliquée sur le rideau. Une campagne d'essais in situ et en laboratoire a été menée pour la caractérisation du sol.

Des prédictions ont été réalisées sur cette expérimentation par de nombreux centres en utilisant la méthode des éléments finis (18 prédictions) et la méthode aux coefficients de réaction ( 25 prédictions). L'analyse des résultats de ces prédictions a montré une nette supériorité de la méthode des éléments finis par rapport à la méthode aux coefficients de réaction [7]. Dans cet article, on propose de présenter brièvement l'expérimentation réalisée et les résultats des prédictions du Laboratoire de Mécanique de Lille, réalisées avec la méthode des éléments finis [4].

\section{2}

\section{Présentation de l'expérimentation}

\section{1}

\section{Dispositif expérimental et programme de chargement [6], [7]}

L'expérimentation a êté effectuée dans le site de Hochstetten proche de Karlsruhe au cours du deuxième trimestre 1993. Elle a comporté le chargement d'un rideau de $7 \mathrm{~m}$ de largeur installé par vibrofonçage jusqu'à $6 \mathrm{~m}$ de profondeur dans un massif de sol limité par deux murs de protection latéraux (Fig. 1a et $1 \mathrm{~b})$. Le programme de chargement a comporté les phases suivantes:

- excavation du massif de sol situé devant le rideau jusqu'à une profondeur de $1,75 \mathrm{~m}$;

(b)

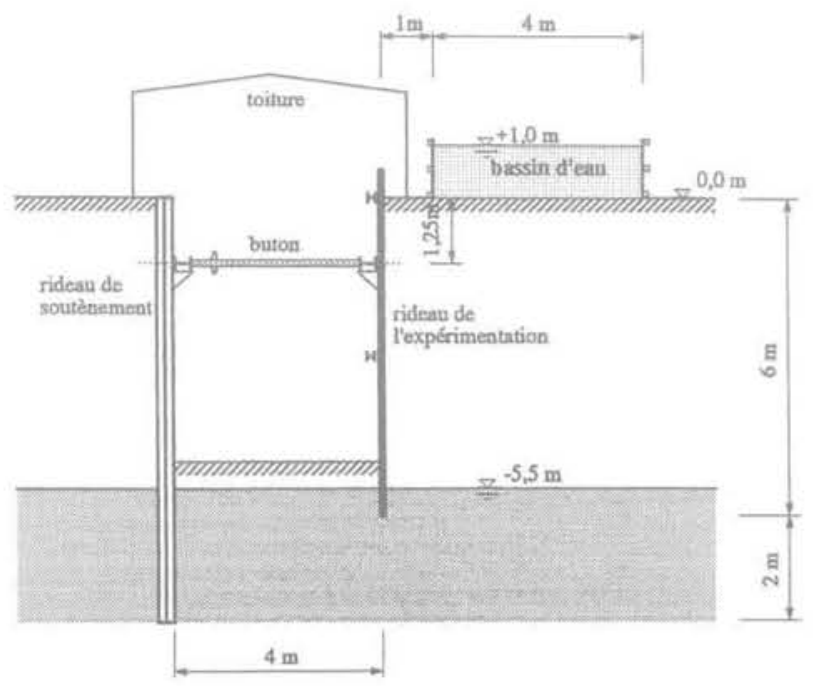

(a) : Vue en plan - Plan view

(b) : Profil transversal - Cross section 


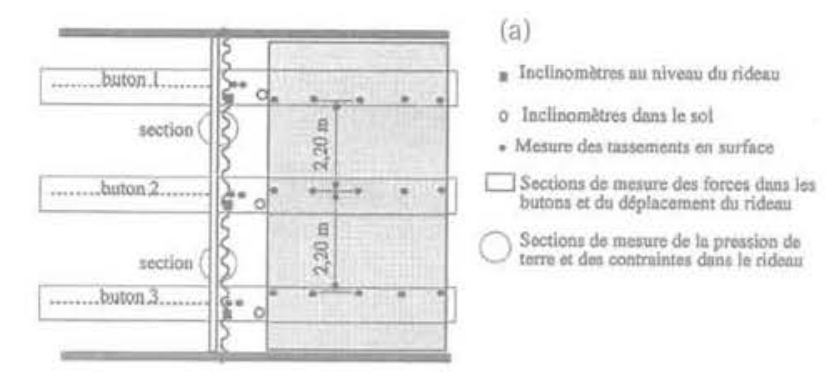

FIG.2 Instrumentation utilisée dans l'expérimentation.

Experimentation set up measurements.

(a): Vue en plan - Plan view

(b) : Profil transversal - Cross section

- installation de trois butons à une profondeur de $1,25 \mathrm{~m}$ et leur préchargement par une force de $11 \mathrm{kN} /$ buton soit de $4,5 \mathrm{kN}$ par mètre de largeur du rideau :

- reprise de l'excavation jusqu'à une profondeur de $5 \mathrm{~m}$; - application d'une pression de $10 \mathrm{kPa}$ sur une zone située entre 1 et $5 \mathrm{~m}$ derrière la tête du rideau,

- réduction de la longueur des butons jusqu'à l'étatlimite qui a été jugé atteint à la stabilisation des forces dans les butons.

\section{2}

\section{Instrumentation}

Les figures $2 \mathrm{a}$ et $2 \mathrm{~b}$ montrent l'instrumentation du rideau et du massif du sol. Deux sections ont été instrumentées par des cellules pneumatiques «Glötzl » pour la mesure de la pression exercée par le sol et par des jauges (DMS) pour la mesure des contraintes de flexion induites dans le rideau. Des inclinomètres ont été installés dans trois sections pour le suivi de la déformée du rideau et pour la mesure du déplacement laté$\mathrm{ral} \mathrm{du} \mathrm{sol} \mathrm{à} 80 \mathrm{~cm}$ derrière le rideau. La déformée du rideau a également été suivie par des mesures géodésiques. Des niveaux ont été utilisés pour la mesure du tassement derrière le mur. Les forces dans les butons ont été mesurées à l'aide des jauges de déformation (DMS); des courbes d'étalonnage ont été établies pour la mesure de ces forces avant l'expérimentation.

Compte tenu de la grande quantité des mesures enregistrées et de leurs dispersions, seules les valeurs moyennes des mesures jugées acceptables ont été utilisées pour analyser le comportement du rideau et pour tester les modèles numériques. Le tableau I donne, pour les phases significatives de chargement, les mesures des pressions appliquées sur les deux phases du rideau, du moment fléchissant, de la déformée et du tassement derrière le rideau.

\section{3}

\section{Propriétés mécaniques du sol}

Des essais en laboratoire et des essais in situ ont été réalisés pour la détermination des propriétés mécaniques du sol. Des analyses sur des échantillons prélevés ont révélé la présence de deux couches de sables mal gradués fins à moyens avec des passages graveleux. La première couche $(0$ à $2 \mathrm{~m})$ est composée d'un

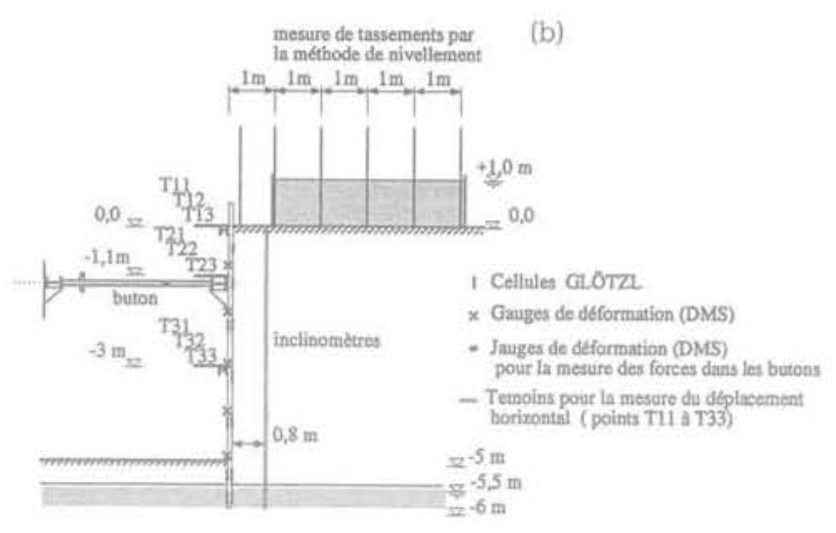

sable silteux, alors que la deuxième est composée d'un sable propre. Des essais triaxiaux ont été effectués sur des échantillons prélevés dans les deux couches. Les résultats de ces essais seront présentés dans la section suivante,

Les essais pressiométriques ont révélé la présence de trois couches dont les caractéristiques pressiométriques sont données dans le tableau II. Les valeurs de la pression limite confirment les résultats des analyses effectuées sur les échantillons prélevés : la pression limite de la première couche $(0,4-0,6 \mathrm{MPa})$ indique un sable argileux ou silteux, les pressions limites des couches inférieures $(0,9-2,3 \mathrm{MPa})$ indiquent la présence d'un sable moyennement compact. Deux essais de plaque avec des cycles ont été effectués. Ils ont donné des modules de déformation de 22 et $27 \mathrm{MPa}$. La figure 3 illustre les résultats de l'un de ces essais.

Du fait que la couche de surface était composée d'un sable fin silteux et que le niveau de la nappe se trouvait à $5,3 \mathrm{~m}$ de profondeur, deux séries d'essais ont été réalisées pour déterminer la valeur de la cohésion capillaire. La première a consisté dans le chargement d'un bloc de sable de $20 \mathrm{~cm}$ de côté et de $30 \mathrm{~cm}$ de hauteur. Les résultats de ces essais ont été analysés avec la méthode d'Hettler [3], qui a donné une cohésion moyenne de $2,7 \mathrm{kPa}$. La seconde série a été effectuée par le chargement d'un bloc excavé sur trois côtés.

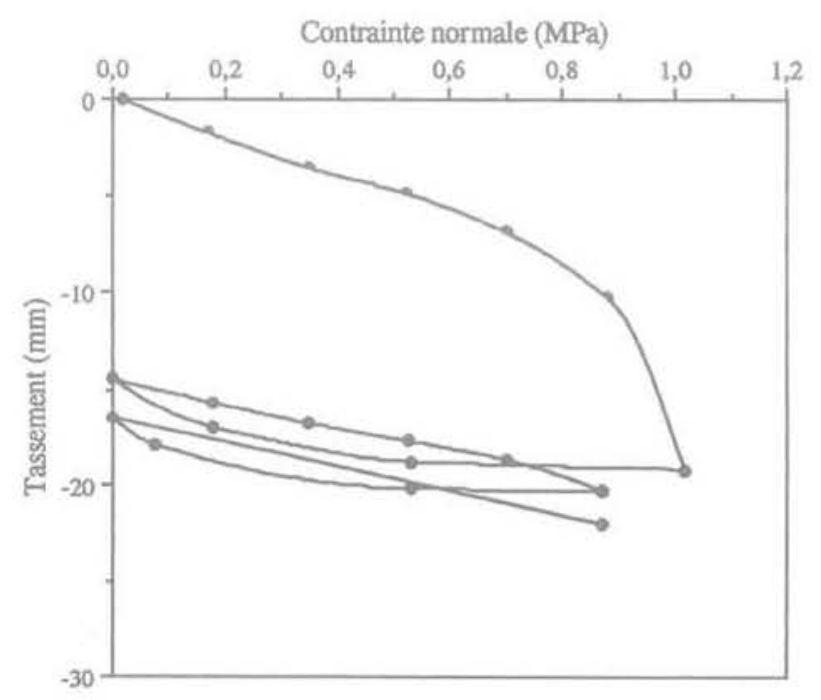

AG. 3 Résultats d'un essai de plaque. Results of a plate loading test. 


\section{\begin{tabular}{l|l|l|l|l|l|l|l|l}
$\mathrm{Z}(\mathrm{m})$ & Phase 1 & Phase 2 & Phase 3 & Phase 4 & Phase 5 & Phase 6 & Phase 7 & Phase 8
\end{tabular} \\ Pression des terres [kPa] (partie gauche, excavation)}

\begin{tabular}{r|r|r|r|r|r|r|r|r}
\hline 2,25 & 31,74 & 52,27 & 48,37 & & & & \\
\hline 4,25 & $-3,23$ & $-3,01$ & $-2,34$ & $-1,78$ & 9,78 & & & \\
\hline 5,25 & 2,65 & 2,58 & 2,27 & 2,49 & 2,82 & 5,36 & 8,50 & 9,09 \\
\hline 5,75 & 15,43 & 15,35 & 15,66 & 15,82 & 16,33 & 18,98 & 20,63 & 18,85 \\
\hline
\end{tabular}

Pression des terres IkPal (partie droite)

\begin{tabular}{r|r|r|r|r|r|r|r|r}
\hline 0,75 & 2,15 & 2,98 & 4,08 & 16,46 & 25,39 & 35,99 & 43,02 & 1,26 \\
\hline 1,25 & 1,05 & 1,62 & 2,98 & 7,57 & 9,48 & 15,15 & 20,39 & 2,09 \\
\hline 2,25 & 32,65 & 29,34 & 26,32 & 6,55 & 3,05 & 3,02 & 6,54 & 10,10 \\
\hline 3,25 & 5,53 & 10,08 & 10,66 & 7,33 & 2,27 & 0,61 & 1,68 & 3,79 \\
\hline 4,25 & 0,00 & 0,00 & 0,00 & 0,00 & 5,42 & 4,63 & 9,40 & 5,28 \\
\hline 5,25 & 3,21 & 3,20 & 3,06 & 3,05 & 3,62 & 3,76 & 5,60 & 4,09 \\
\hline 5,75 & 17,86 & 17,95 & 17,98 & 18,03 & 18,72 & 21,81 & 25,48 & 34,78 \\
\hline
\end{tabular}

Moment fléchissant $[\mathrm{kNm} / \mathrm{m}]$

\begin{tabular}{r|r|r|r|r|r|r|r|r}
\hline 1,00 & $-0,89$ & $-1,25$ & $-2,66$ & $-2,55$ & $-3,58$ & $-4,41$ & $-5,06$ & $-0,09$ \\
\hline 2,00 & $-0,55$ & $-2,26$ & $-2,12$ & 0,26 & 1,37 & 1,53 & 1,70 & 4,67 \\
\hline 3,00 & $-0,08$ & $-0,75$ & $-0,93$ & $-1,14$ & 1,14 & 2,20 & 2,76 & 3,41 \\
\hline 4,00 & $-0,05$ & $-0,03$ & 0,02 & $-0,56$ & $-1,56$ & 0,37 & 0,78 & $-2,83$ \\
\hline 5,00 & $-0,02$ & $-0,02$ & 0,06 & 0,11 & $-0,09$ & $-1,18$ & $-1,70$ & $-7,33$ \\
\hline
\end{tabular}

Déplacement latéral [mm]

\begin{tabular}{c|c|c|c|c|c|c|c|c}
\hline 0,00 & 2,45 & 7,75 & 8,25 & 7,55 & 7,05 & 5,55 & 5,15 & 29,65 \\
\hline 0,60 & 1,57 & 5,95 & 6,18 & 5,65 & 5,61 & 4,33 & 4,23 & 26,67 \\
\hline 1,10 & 0,95 & 4,33 & 4,37 & 4,18 & 4,35 & 3,37 & 3,55 & 24,25 \\
\hline 1,60 & 0,34 & 2,61 & 2,65 & 2,85 & 3,39 & 2,78 & 3,06 & 21,58 \\
\hline 2,10 & 0,05 & 1,25 & 1,21 & 1,90 & 2,63 & 2,72 & 3,11 & 19,01 \\
\hline 2,60 & $-0,17$ & 0,31 & 0,32 & 1,14 & 2,17 & 2,88 & 3,36 & 16,28 \\
\hline 3,10 & $-0,17$ & 0,05 & 0,04 & 0,62 & 1,72 & 2,99 & 3,40 & 13,36 \\
\hline 3,60 & $-0,17$ & $-0,05$ & $-0,04$ & 0,19 & 1,11 & 2,67 & 3,11 & 9,98 \\
\hline 4,10 & $-0,17$ & $-0,10$ & 0,00 & 0,00 & 0,45 & 2,14 & 2,43 & 6,50 \\
\hline 4,60 & 0,11 & 0,05 & 0,09 & 0,14 & 0,20 & 1,49 & 1,65 & 3,68 \\
\hline 5,10 & 0,11 & 0,05 & 0,09 & 0,09 & 0,10 & 0,64 & 0,78 & 1,31 \\
\hline 5,60 & 0,00 & 0,00 & 0,00 & 0,00 & 0,00 & 0,00 & 0,00 & 0,00 \\
\hline 6,00 & 0,00 & 0,00 & 0,00 & 0,00 & 0,00 & 0,00 & 0,00 & 0,00 \\
\hline
\end{tabular}

Déplacement vertical [mm]

\begin{tabular}{c|c|c|c|c|c|c|c|c}
\hline $\begin{array}{c}\text { Distance } \\
\text { du rideau } \\
(\mathrm{m})\end{array}$ & & & & & & & & \\
\hline 0,00 & $-8,00$ & $-3,40$ & $-0,63$ & $-3,83$ & $-3,60$ & $-3,00$ & $-3,57$ & $-16,33$ \\
\hline 1,00 & $-1,00$ & $-0,67$ & $-0,80$ & $-1,07$ & $-1,60$ & $-2,17$ & $-2,80$ & $-7,23$ \\
\hline 2,00 & $-1,67$ & $-0,43$ & $-0,43$ & $-0,67$ & $-1,33$ & $-1,70$ & $-2,83$ & $-5,77$ \\
\hline 3,00 & $-0,33$ & $-0,27$ & $-0,43$ & $-0,77$ & $-1,03$ & $-1,13$ & $-2,47$ & $-7,23$ \\
\hline 4,00 & $-1,00$ & $-0,17$ & $-0,30$ & $-0,60$ & $-0,70$ & $-0,90$ & $-0,90$ & $-2,83$ \\
\hline 5,00 & $-2,67$ & $-0,30$ & $-0,70$ & $-1,07$ & $-1,17$ & $-1,30$ & $-1,30$ & $-2,47$ \\
\hline
\end{tabular}

Phase 1: Excavation jusqu'à $1 \mathrm{~m}$

Phase 2: Excavation jusqu'á $1.75 \mathrm{~m}$

Phase 3 : Installation du buton

Phase 4: Excavation jusqu'à $3 \mathrm{~m}$

Phase 5 : Excavation jusqu'à $4 \mathrm{~m}$

Phase 6: Excavation jusqu'à $5 \mathrm{~m}$

Phase 7 : Application d'une pression à la surface

Phase 8: État-limite 


\begin{tabular}{c|c|c}
\hline Cote $(\mathrm{m})$ & $\mathrm{E}_{0}(\mathrm{MPa})$ & $\mathrm{P}_{1}(\mathrm{MPa})$ \\
\hline $0-2,3$ & $4,4-11,4$ & $0,4-0,6$ \\
\hline $2,3-4$ & $6,3-14$ & $0,9-1,1$ \\
\hline $4-10$ & $13,6-18,9$ & $1,7-2,3$ \\
\hline
\end{tabular}

L'analyse des résultats obtenus a donné une cohésion moyenne de $4,0 \mathrm{kPa}$.

\section{3}

\section{Prédictions numériques}

Les prédictions ont été effectuées en utilisant un modèle élastique-parfaitement plastique implanté dans le programme de calcul par éléments finis PECPLAS [4]. L'expérimentation a été modélisée avec l'hypothèse des déformations planes dans une section courante. Nous allons, ci-après, présenter brièvement le modèle utilisé, la démarche suivie pour la modélisation et les résultats obtenus.

\section{1}

\section{Modélisation du comportement du sol}

Le comportement du sol a été décrit par un modèle élastique-parfaitement plastique utilisant le critère de Mohr-Coulomb et une règle d'écoulement non associée. La partie élastique est supposée linéaire et isotrope avec deux paramètres : le module d'Young (E) et le coefficient de Poisson (v). La fonction de charge et le potentiel de plasticité sont écrits en conditions de déformations planes:

$$
\begin{aligned}
& f=\sigma_{1}-\sigma_{3}+\left(\sigma_{1}+\sigma_{3}\right) \sin (\varphi)-2 \operatorname{Cos}(\varphi) \\
& g=\sigma_{1}-\sigma_{3}+\left(\sigma_{1}+\sigma_{3}\right) \sin (\psi)
\end{aligned}
$$

$\sigma_{1}$ et $\sigma_{3}$ sont respectivement les contraintes principales majeure et mineure.

$C$, $\varphi$ et $\psi$ désignent respectivement la cohésion, l'angle de frottement et l'angle de dilatance du sol.

Les essais triaxiaux ont été utilisés pour la détermination des paramètres mécaniques. Des difficultés ont été rencontrées dans la détermination des paramètres élastiques à cause de la grande dispersion des résultats des essais triaxiaux. Compte tenu du niveau des contraintes au cours de l'expérimentation, nous avons privilégié les essais réalisés aux fiables confinements ( 50 et $100 \mathrm{kPa}$ ). Ces essais ont donné $\mathrm{E}=20 \mathrm{MPa}$ pour la première couche, et $\mathrm{E}=30 \mathrm{MPa}$ pour la deuxième couche. Le coefficient de Poisson a été supposé constant dans tout le massif $(v=0,3)$. Les paramètres élastiques déterminés pour la première couche ont été vérifiés sur les résultats des essais de plaque: avec un module d'Young de $20 \mathrm{MPa}$ et un coefficient de Poisson de 0,3 , on obtient un module œdométrique de $27 \mathrm{MPa}$ qui est la valeur moyenne des modules obtenus avec les deux essais de plaque (22 et $32 \mathrm{MPa}$ ).

La détermination des paramètres de plasticité (angle de frottement, cohésion et angle de dilatance) à partir
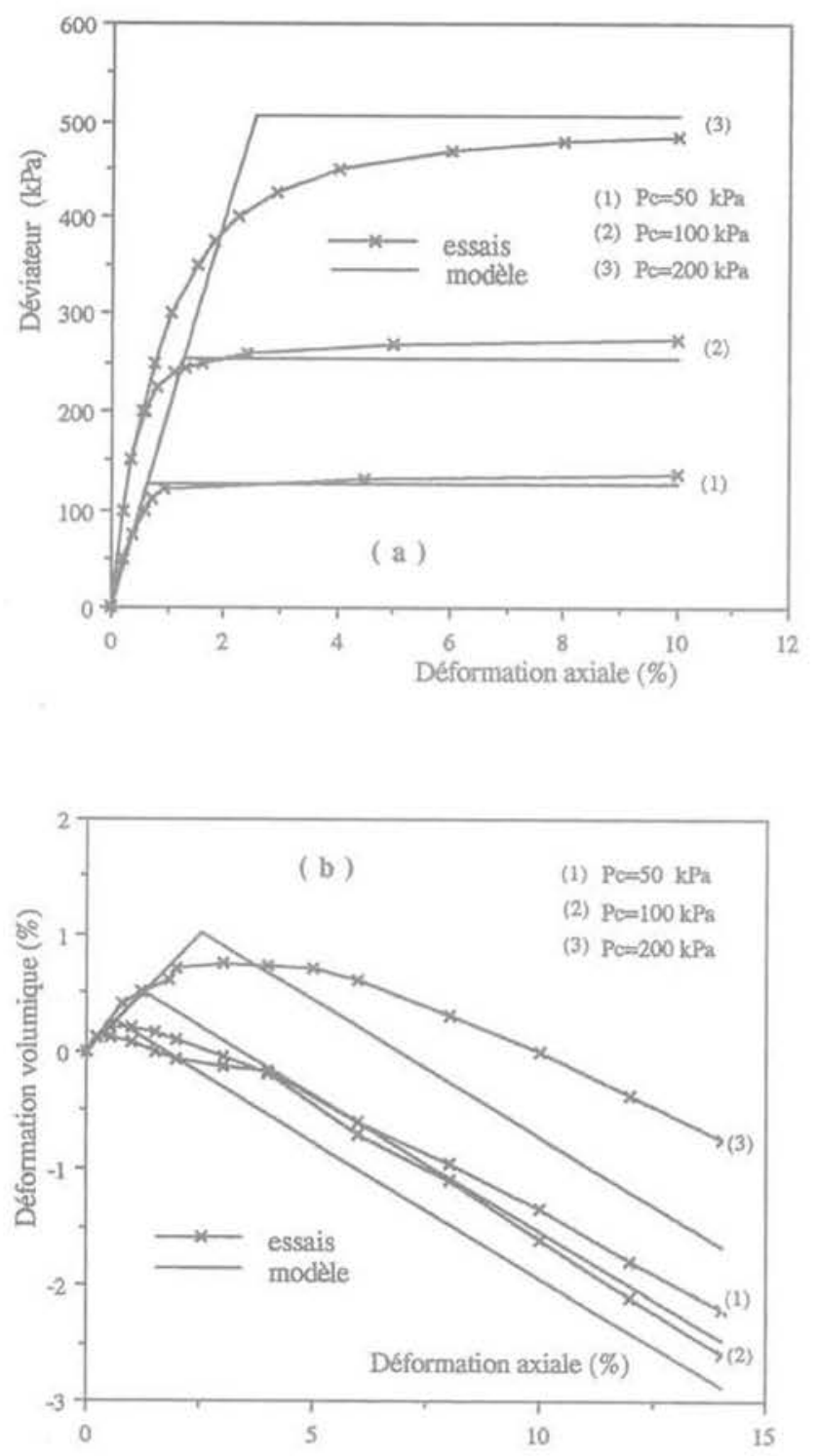

FG.4 Résultats des essais triaxiaux et de leur simulation avec le modèle de Mohr-Coulomb non associé.

(a) : Déviateur

(b) : Déformation volumique Results of triaxial tests and their simulation using non associated Mohr-Coulomb constitutive model.

(a) : Deviator

(b) : Volumetric strain

des essais triaxiaux n'a pas posé de difficultés. Ces essais ont donné pour la première couche $\varphi=34^{\circ}, \mathrm{C}=0$ et $\psi=6^{\circ}$, et pour la deuxième couche $\varphi=40^{\circ}$, $\mathrm{C}=0$ et $\psi=12^{\circ}$. Les figures $4 \mathrm{a}$ et $4 \mathrm{~b}$ illustrent les résultats des essais triaxiaux et leur simulation avec le jeu de paramètres déterminé ci-dessus. On peut noter une reproduction correcte de la résistance du sol et de l'évolution de la déformation volumique.

Compte tenu de la présence d'éléments fins dans le sol et du niveau de la nappe (à $5,3 \mathrm{~m}$ de la surface du sol) une cohésion d'origine capillaire a été considérée dans la modélisation. Étant donné les valeurs mesurées pour cette cohésion $(\mathrm{C}=2,6$ et $4,0 \mathrm{kPa}$ ), nous avons retenu une cohésion moyenne de $3 \mathrm{kPa}$. Le tableau III récapitule le jeu de paramètres utilisé dans les prédictions numériques. 


\begin{tabular}{c|c|c|c|c|c}
\hline Couche & E(MPa) & $v$ & $\varphi\left({ }^{\circ}\right)$ & $\begin{array}{c}\text { Ccapillaire } \\
\text { (kPa) }\end{array}$ & $\psi\left({ }^{\circ}\right)$ \\
\hline$z<2 m$ & 20 & 0,3 & 34 & 3 & 6 \\
\hline$z>2 m$ & 30 & 0,3 & 40 & 3 & 12 \\
\hline
\end{tabular}

Le comportement du rideau a été supposé élastiquelinéaire avec une rigidité à la flexion $\mathrm{EI}=2032 \mathrm{MPa} \cdot \mathrm{m}^{2} / \mathrm{m}$.

3.2

\section{Modélisation de l'expérimentation}

Le sol et le rideau ont été modélisés en utilisant des éléments quadrilatères à 8 nœuds. Ces éléments permettent de décrire les effets de flexion dans le rideau. Le maillage utilisé est montré dans la figure 5 . Il comporte 240 éléments quadrilatères à 8 nœuds et 783 nœuds. Il est raffiné au voisinage du rideau pour la prise en compte de la variation des contraintes dans cette région et pour une bonne évaluation de l'action du sol sur le rideau.

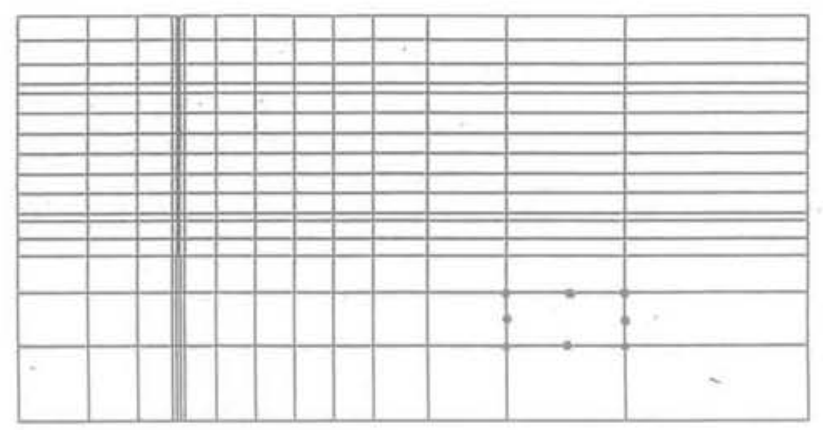

FIG. 5 Maillage utilisé : 240 éléments quadrilatères à 8 nœuds et 783 nœuds. Finite element nesh used in numerical predictions.

La distribution des contraintes initiales est supposée de type œdométrique avec un coefficient de poussée latérale au repos $K_{0}=0,4$. Cette hypothèse n'est pas en accord avec les mesures in situ qui montrent que l'installation du rideau induit une modification sensible des contraintes au voisinage du rídeau (Fig. 6). Néanmoins, cette hypothèse a été maintenue, car la prise en compte de l'installation du rideau constitue un problème fort complexe de la modélisation en géomécanique dont la vérification n'est pas l'objet du présent travail. Ajoutons à cela que l'installation du rideau affecte essentiellement la distribution des contraintes dans son voisinage, alors que le chargement, induit par l'excavation, implique une masse plus importante du sol où la distribution des contraintes initiale peut être raisonnablement supposée de type œdométrique.

L'excavation a été modélisée par une désactivation des éléments du sol se trouvant dans la zone à excaver. Le buton, assumé infiniment rigide, a été modélisé par un déplacement imposé au nœud correspondant à sa position. Pendant les trois premières phases de char-

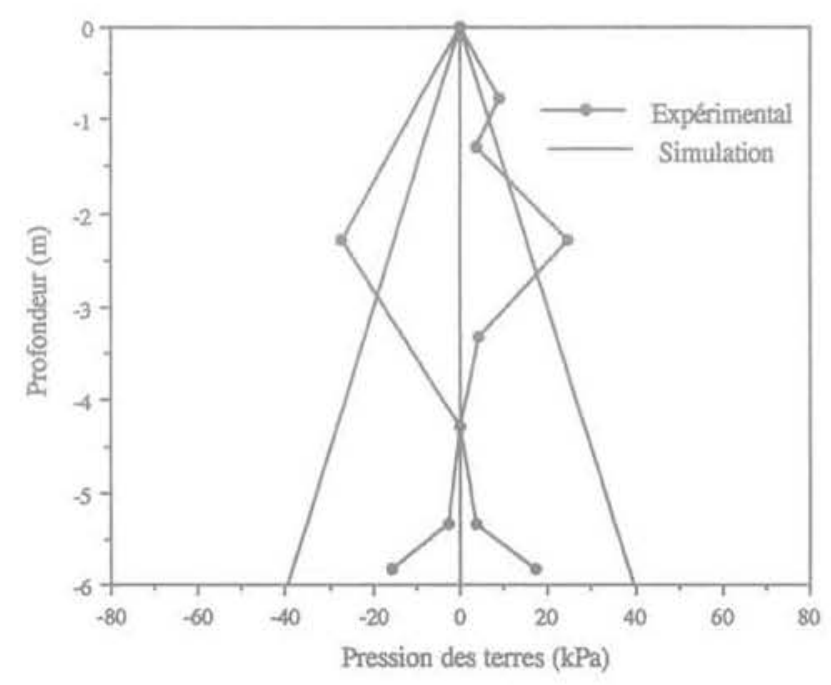

FIG.6 Distribution de la pression de terre initiale appliquée au rideau.

Initial earth pressure applied on the sheet pile.

gement, le déplacement horizontal de ce nœud a été bloqué, la dernière phase de chargement a été modélisée par un déplacement horizontal croissant jusqu'à la stabilisation de la force dans les butons. La figure 7 montre l'évolution de cette force en fonction du déplacement imposé. On constate que la force tend à se stabiliser à partir d'un déplacement de l'ordre de $3 \mathrm{~cm}$ et pour un niveau de force de l'ordre de $8,3 \mathrm{kN} / \mathrm{m}$. Cet état a été considéré comme l'état-limite dans la modélisation.

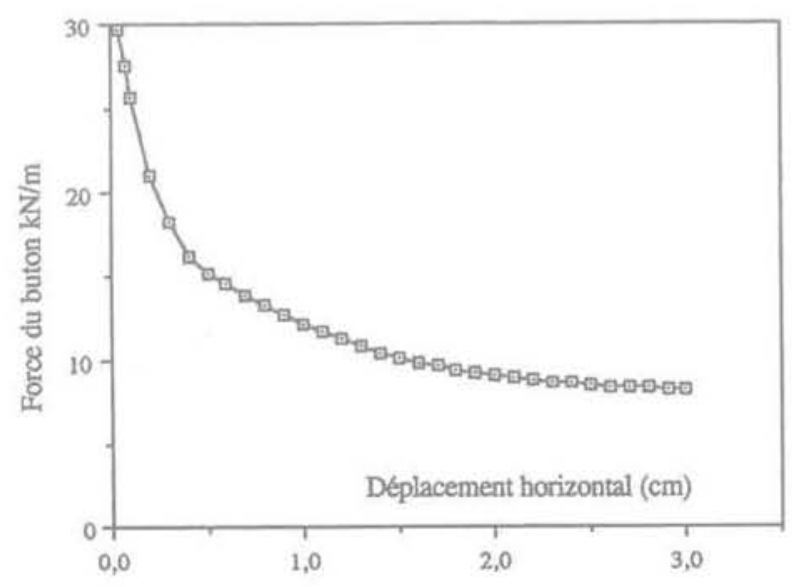

FG.7 Evolution des forces dans les butons au cours de la réduction de leur longueur. Evolution of struts forces during the reduction of their lergth. 


\section{Présentation des résultats des prédictions}

Compte tenu de la grande quantité de mesures, la confrontation des prédictions numériques aux résultats de l'expérimentation a porté sur quatre phases significatives du chargement, à savoir :

- excavation devant le rideau jusqu'à une profondeur de $4 \mathrm{~m}$ (phase I) ;

- excavation jusqu'à la profondeur maximale $\mathrm{Z}=5 \mathrm{~m}$ (phase II);

- application de la pression en surface (phase III) ;

- stabilisation des forces dans les butons (état-limite) (phase IV).

\subsection{1.}

\section{Forces dans les butons}

Le tableau IV donne les valeurs des forces dans les butons mesurées au cours de l'expérimentation et obtenues par les prédictions numériques. On constate un bon accord entre les prédictions et l'expérimentation pour les deuxième et troisième phases. Pour la première phase, les prédictions numériques sous-estiment les forces dans les butons. Cela peut être attribué à la non prise en compte de l'installation du rideau qui a eu pour effet une augmentation des pressions des terres dans sa partie supérieure (Fig. 6). Pour la dernière phase, on note que les prédictions numériques surestiment la force dans le buton. Cet écart peut être, en partie, attribué aux difficultés rencontrées pour assurer une bonne convergence en fin du calcul.

\section{3 .2}

\section{Déformée du rideau}

Les figures $8 \mathrm{a}$ et $8 \mathrm{~b}$ montrent les courbes de la déformée du rideau obtenues avec l'expérimentation et les prédictions numériques. On constate que l'expérjmentation et les prédictions numériques sont en bon accord pour les trois premières phases, en particulier dans la partie supérieure de rideau $(Z<4 \mathrm{~m})$ où la déformée est marquée par une rotation autour de la position du buton; dans la partie inférieure, on note que l'expérimentation donne un déplacement nul alors que les prédictions numériques donnent un déplacement de l'ordre de $2 \mathrm{~mm}$. Pour la dernière phase de chargement, on note un désaccord important entre l'expérimentation et les prédictions : la déformée obtenue expérimentalement est marquée par une rotation autour du centre du rideau alors que la déformée obtenue par les prédictions numériques est marquée dans la partie supérieure $(\mathrm{Z}<3 \mathrm{~m})$ par une rotation autour de la position du buton, et dans la partie inférieure par une rotation autour du centre de cette partie.
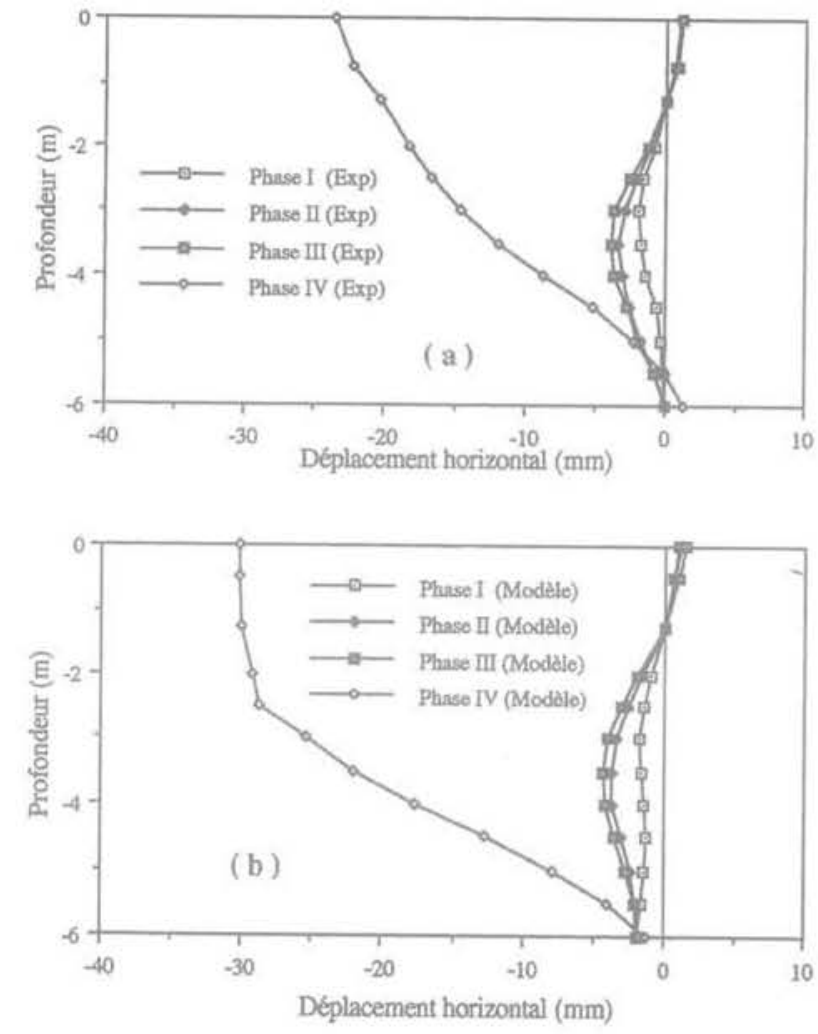

FiG.8 Évolution de la déformée du rideau. (a) : Expérimentale (b) : Prédictions numériques

Evolution of the deflection of the sheet pile.

(a) : Experimental

(b) : Numerical predictions

\begin{abstract}
$3=3.5$
Pressions des terres

Les figures $9 a$ et $9 b$ montrent les pressions des terres appliquées sur le rideau obtenues avec l'expérimentation et les simulations numériques. On constate que pour les trois premières phases de chargement les prédictions numériques et les résultats expérimentaux indiquent une augmentation de la pression des terres dans la partie supérieure du rideau et une diminution de celle-ci dans la partie inférieure. Ces évolutions correspondent respectivement à une mobilisation partielle de la butée et de la poussée des terres. On note cependant que le modèle sous-estime d'une manière sensible la pression des terres au voisinage du buton. Dans la partie inférieure, on note que le modèle surestime les pressions appliquées sur les deux côtés du rideau, en
\end{abstract}

TABLEAUN Forces dans les butons obtenues par l'expérimentation et la modélisation numérique.

\begin{tabular}{l|c|c|c|c}
\cline { 2 - 4 } & $\begin{array}{c}\text { F(kN/mi) } \\
\text { Phase I }\end{array}$ & $\begin{array}{c}\text { F (kN/mi) } \\
\text { Phase II }\end{array}$ & $\begin{array}{c}\text { F(kN/ml) } \\
\text { Phase II }\end{array}$ & $\begin{array}{c}\text { F(kN/mi) } \\
\text { Phase IV }\end{array}$ \\
\cline { 2 - 5 } Expérimentale & 21,3 & 28,6 & 33,7 & 4,2 \\
\hline Prédiction & 16,3 & 26,9 & $32,7 \mathrm{kN}$ & 8,3 \\
\hline
\end{tabular}



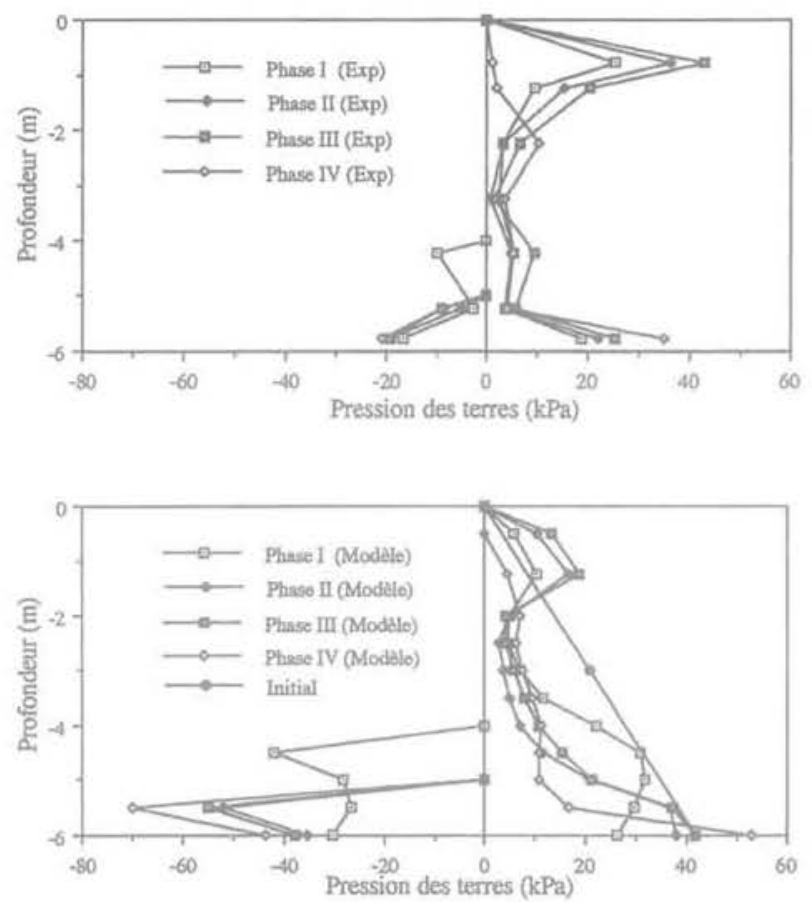

FG.9 Évolution de la pression des terres. (a) : Expérimentale (b) : Prédictions numériques

Evolution of earth pressure applied on the sheetpile wall.

(a) : Experimental

(b) : Numerical predictions

particulier dans la partie avant où on note une mobilisation importante de la butée alors que l'expérimentation indique une faible mobilisation de celle-ci. Pour la dernière phase de chargement, on note un bon accord entre les prédictions et l'expérimentation sur la pression appliquée sur la face arrière du rideau, mais une surestimation par la modélisation de la pression de butée appliquée sur la face avant.

\subsection{4.}

\section{Moment de flexion}

Les figures $10 \mathrm{a}$ et $10 \mathrm{~b}$ montrent les courbes du moment fléchissant obtenues expérimentalement et par la modélisation. On note que pendant les trois premières phases de chargement, l'expérimentation et les prédictions donnent un moment fléchissant négatif aux extrémités du rideau et un moment positif dans la partie centrale. Au niveau quantitatif, on note que les prédictions numériques surestiment ce moment dans les
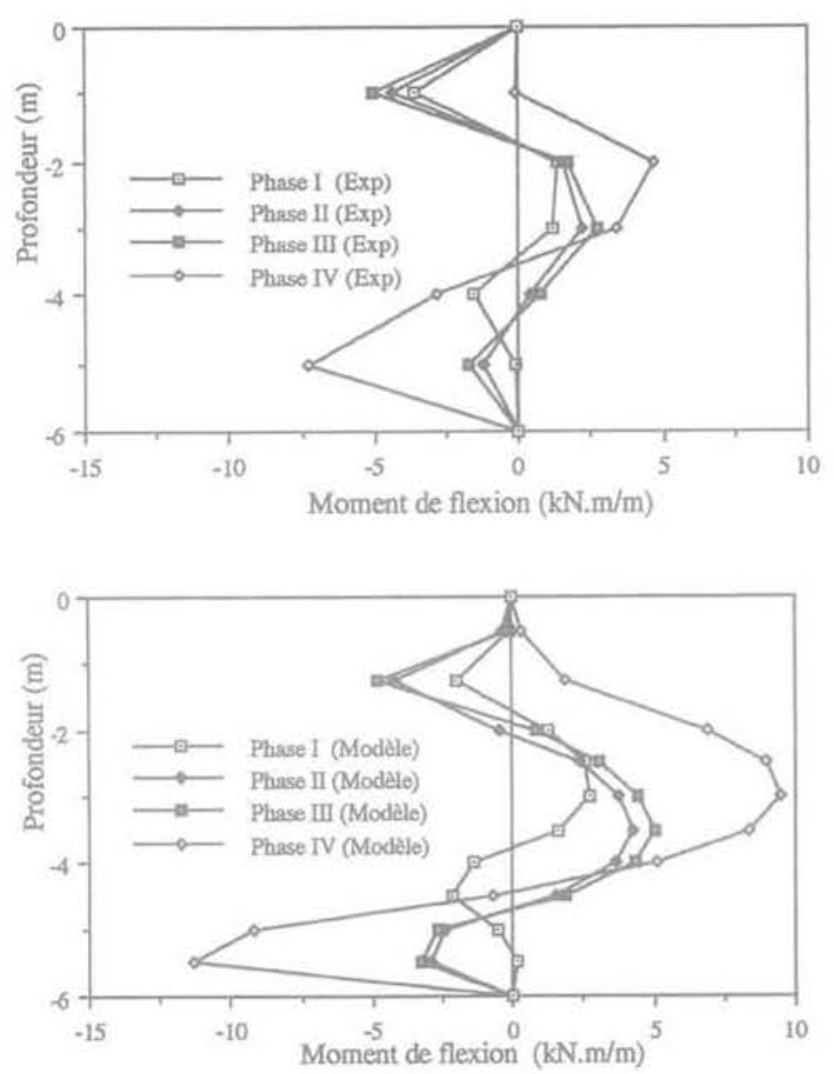

FG. 10 Évolution du moment de flexion.

\section{(a) : Expérimentale}

(b) : Prédictions numéríques

Evolution of bending moment.

(a) : Experimental

(b) : Numerical predictions

parties centrale et inférieure du rideau. Pour la quatrième phase, on note un accord qualitatif entre l'expérimentation et la modélisation, mais cette dernière donne des valeurs élevées du moment par rapport à celles mesurées au cours de l'expérimentation.

\section{Étude paramétrique}

Compte tenu des incertitudes qu'on peut avoir sur la détermination des paramètres des modèles de sols, en particulier les paramètres élastiques, une étude a été

TABLEAUY Influence de la perturbation des paramètres sur les forces dans les butons.

\begin{tabular}{l|c|c|c|c}
\cline { 2 - 5 } & $\begin{array}{c}\text { F(kN/ml) } \\
\text { Phase I }\end{array}$ & $\begin{array}{c}\text { F (kN/ml) } \\
\text { Phase II }\end{array}$ & $\begin{array}{c}\text { F (kN/ml) } \\
\text { Phase III }\end{array}$ & $\begin{array}{c}F(\mathrm{kN} / \mathrm{ml}) \\
\text { Phase IV }\end{array}$ \\
\cline { 2 - 5 } Rérérences & 16,3 & 26,9 & 32,7 & 8,3 \\
\hline$+50 \% \mathrm{E}$ & 16,1 & 26,7 & 32,7 & 10,3 \\
\hline$+10 \% \phi$ & 15,5 & 25 & 30,3 & 6,2 \\
\hline $\mathrm{C}=1 \mathrm{kPa}$ & 19,9 & 32,8 & 39,7 & 20,4 \\
\hline
\end{tabular}



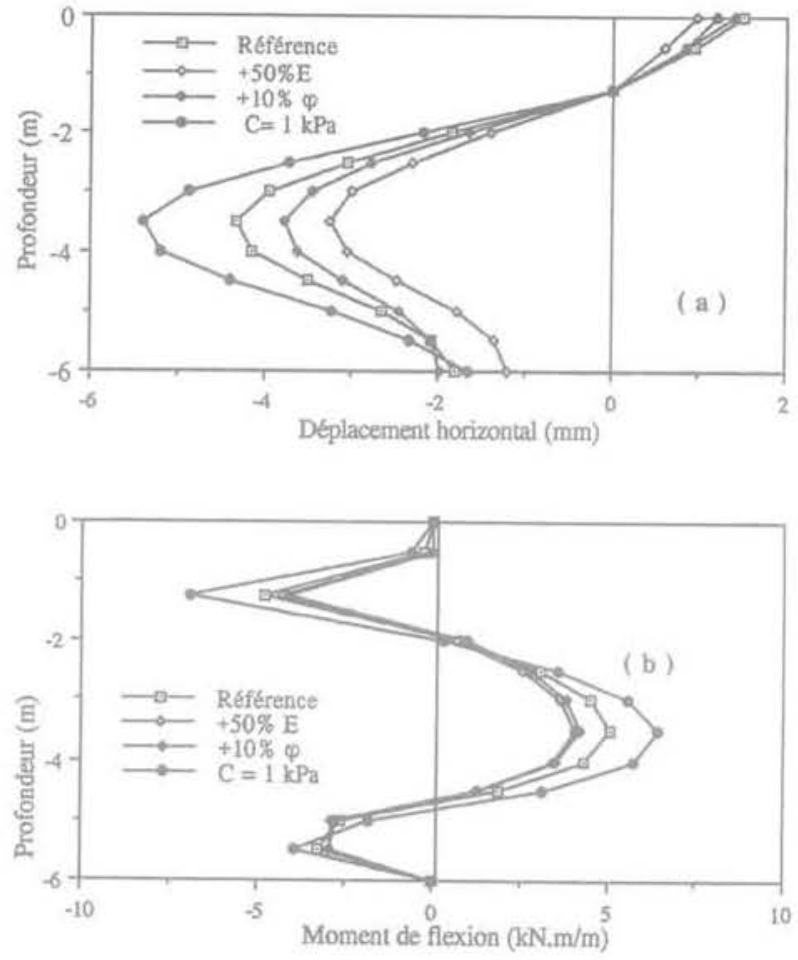

FH.11 Influence de la perturbation des paramètres mécaniques sur les prédictions numériques.

(a) : Déformée du rideau (b) : Moment de flexion Influence of a perturbation of constitutive parameters on the sheet pile behaviour. (a) : Sheetpile deflection

(b) : Bending moment

réalisée pour analyser l'influence de la perturbation de certains paramètres sur la simulation numérique de l'expérimentation. Dans un premier temps, cette étude a comporté une analyse de l'influence de la perturbation du module d'Young et l'angle de frottement sur les prédictions numériques. Les résultats de cette étude sont illustrés dans les figures $11 \mathrm{a}-11 \mathrm{~b}$ et le tableau V. On constate qu'une augmentation de $50 \%$ du module d'Young induit une augmentation importante de la déformée du rideau (de l'ordre de $30 \%$ pour la valeur maximale) et du moment fléchissant (de l'ordre de $20 \%$ pour la valeur maximale), mais elle n'influence que légèrement les forces dans les butons. Une augmentation de $10 \%$ de l'angle de frottement induit une diminution de $15 \%$ de la valeur maximale de la déformée, de $20 \%$ de la valeur maximale du moment fléchissant et de $8 \%$ de la force dans les butons. Ces résultats montrent la nécessité d'une bonne estimation de ces paramètres, en particulier du module d'Young dont la détermination à partir des essais triaxiaux conventionnels peut comporter de grandes incertitudes.

Afin d'étudier l'influence de la cohésion capillaire sur le comportement du rideau, une simulation a été effectuée en considérant une faible valeur de cette cohésion $(\mathrm{C}=1 \mathrm{kPa})$. Les résultats obtenus sont illustrés dans les figures $11 \mathrm{a}-11 \mathrm{~b}$ et le tableau V. On constate que cette diminution induit une augmentation moyenne de la déformée du rideau (de l'ordre de $25 \%$ pour la valeur maximale) et du moment fléchissant (de l'ordre de $30 \%$ ppour la valeur maximale) et une augmentation très sensible des forces dans les butons (de l'ordre de $150 \%$ ). Ces résultats montrent le rôle important que joue la cohésion capillaire dans le calcul en déformation des rideaux.

\section{5}

\section{Conclusion}

Dans cet article nous avons présenté les résultats d'une expérimentation en vraie grandeur sur un rideau de palplanche butonné qui a été chargé par excavation et pression en surface et a été amené à l'état-limite par réduction des forces de butonnage. La quantité et la qualité des mesures réalisées sur cet ouvrage en font un bon exemple pour la vérification des modèles utilisés dans le calcul des rideaux de soutènement.

Une prédiction de cette expérimentation a été réalisée à l'aide d'une modélisation par éléments finis avec un modèle élastique - parfaitement plastique utilisant le critère de Mohr-Coulomb non associé. Cette prédiction a montré que ce modèle décrit correctement le comportement du rideau dans les phases de fonctionnement, à savoir l'excavation et le chargement en surface. Des difficultés ont été rencontrées pour le calcul de la première phase de chargement à cause de la négligence de la perturbation induite par l'installation du rideau. Une étude paramétrique a montré que les prédictions numériques sont particulièrement sensibles au module d'Young et à la cohésion capillaire. Du fait que ces paramètres sont très sensibles au remaniement, il est recommandé de les déterminer à partir d'essais in situ.

\section{Bibliographie}

[1] Balay J., Frank R., Harfouche L. - Programme DENEBOLA pour le calcul des soutènements par la méthode des modules de réactions, Bulletin de Liaison des LPC, 120, juillet-aoút, p. 3-12, 1982.

[2] Balay J, - Recommandations pour le choix des paramètres de calcul des écrans de soutènement par la méthode aux modules de réaction, Note d'Information Technique du Laboratoire Central des Ponts et Chaussées, 1984.

[3] Hettler A. - Setzungen von Einzelfunda- menten auf sand, Bautechnik 6, p. 189 . 197, 1985.

[4] Shahrour 1-PECPLAS: A finite element software for the resolution of earthwork problems, Actes du Colloque International Gèotechnique-Informatique, Paris, Presses de I'ENPC, p. 327 334, 1992.

[5] Shahrour 1., Ghorbanbeigi S. - Prediction of the sheetpile wall field test of Karlsruhe using an elastoplastic finite element modelling. Workshop Sheet
Pile Test Karlsruhe, Delft University, Holland, October, 1994.

[6] Wolffersdorff P.-A. von - Feldversuch an einer Spundwand in Sandboden Versuchsergbnisse und Prognosen, Geotechnik 17, p. 73-83, 1994.

[7] Wolffersdorff P.-A. von - Results of the field test and evaluation of the predictions and subsequent calculations, Workshop Sheet Pile Test Karlsruhe, Delft University, Holland, October 1994. 